\title{
High-performance sensors based on organic electrochemical transistors with various gate electrodes
}

\author{
Shiwei Lin ${ }^{*}$, Jianjun Liao \\ State Key Laboratory of Marine Resource Utilization in South China Sea, Hainan University, Haikou \\ 570228, PR China \\ *Corresponding author's e-mail address: linsw@hainu.edu.cn
}

\begin{abstract}
:
Organic electrochemical transistors (OECTs) show promising applications in various chemical and biological sensors. The advantages of OECT-based sensors include low cost, easy fabrication, low operational voltage, mechanical flexibility and good biocompatibility. In this paper, we report three high-performance OECT-based sensors, including diatom, glucose and chemical oxygen demand sensors. Various gate electrodes, including $\mathrm{Au}, \mathrm{TiO}_{2}$ nanotube arrays and $\mathrm{Pt}$ nanoparticles modified $\mathrm{TiO}_{2}$ nanotube arrays, have been selected and modified according to different demands. The sensing mechanisms and the perspective are described. In terms of the diverse materials selection and easy surface modification of the gate electrodes, it is expected that these kinds of OECT-based sensors may have more important applications in chemical and biological sensing.
\end{abstract}

Key words: Organic electrochemical transistor, gate electrode, chemical sensor, biosensor, PEDOT:PSS

\section{Introduction}

Organic thin film transistors (OTFTs) are attracting a great deal of interest for sensor applications due to their simple electrical readout, inherent signal amplification, straightforward miniaturization, and facile incorporation into arrays and circuits. Within the family of OTFT-based sensors, organic electrochemical transistors (OECTs) have been extensively studied for their particular advantages, including easy fabrication, low operational voltage $(\sim 1 \mathrm{~V})$, good biocompatibility, low cost and mechanical flexibility [1]. To date, OECTs have been successfully used in sensing different species of analysts, including ions, glucose, proteins, DNA detection, as well as recording transepithelial ion transport.

OECTs operation relies on electrochemical doping/de-doping of an organic semiconductor film in contact with an electrolyte. These devices can operate in liquid environment at low voltages, which make them particularly suitable for biological and chemical detection. Take an OECT-based glucose sensor for example. Poly(3,4-ethylenedioxythiophene): poly(styrenesulfonic acid) (PEDOT:PSS) was used as an active layer, while the enzyme glucose oxidase could be floating in the buffered solution, or fixed on the organic semiconductor, or even fixed on the gate electrode during the measurements. The sensing properties are closely linked with and can be modulated by these configurations, which is more convenient in real applications.

In this paper, we report three high-performance OECT-based sensors recently developed in our group using PEDOT:PSS as the active layer. Various gate electrodes have been selected and modified according to different demands. A Au electrode was immersed into the electrolyte as the gate electrode, which has been successfully used for the detection of marine diatoms in the seawater medium. Using $\mathrm{TiO}_{2}$ nanotube arrays-based gate electrodes, we present another kind of highly selective and sensitive glucose sensors. Furthermore, we report a novel and miniaturized OECT device using $\mathrm{Pt}$ nanoparticles modified $\mathrm{TiO}_{2}$ nanotube arrays (Pt-NPs/TNTAs) as the gate electrode for the photoelectrochemical determination of chemical oxygen demand (COD). The corresponding sensing mechanisms have also been discussed respectively. 
Biosensor with Au gate electrode for detecting diatoms in seawater medium

OECTs with PEDOT:PSS as the active layer have been successfully used as diatoms sensors for detecting the marine diatoms in the seawater medium for the first time [2], where Au electrode was immersed into the electrolyte as the gate electrode. OECTs showed stable performance and excellent biocompatibility in seawater medium. The sensors can be used to detect two typical diatoms: Navicula sp. and Amphiprore sp. Maximum gate offset voltages, about $60 \mathrm{mV}$ for Navicula sp. and $28 \mathrm{mV}$ for Amphiprore sp., were observed after the attachment of diatoms. The transfer curves of OECT device shifted to higher gate voltage after the capture of diatoms, which can be attributed to the electrostatic interaction between diatoms and the PEDOT:PSS layer. The devices can detect Navicula sp. and Amphiprore sp. concentrations down to 100 cell $/ \mathrm{mL}$ and 400 cell $/ \mathrm{mL}$, respectively.

\section{Glucose sensors using $\mathrm{TiO}_{2}$ nanotube arrays-based gate electrodes}

TNTAs electrodes have been successfully used as the gate electrodes of the OECT-based glucose sensors for the first time [3]. The sensing performance of the OECT using TNTAs-based gate electrode is comparable to or better than those of the reported OECTs using Pt-based gate electrodes. Highly sensitive and selective OECT devices can be obtained by modifying TNTAs electrodes with Pt nanoparticles and enzyme (glucose oxidase). A linear range between the channel current and the logarithm of glucose concentration is exhibited from $100 \mathrm{nM}$ to $5 \mathrm{mM}$, and the detection limit is down to $100 \mathrm{nM}$, which is three orders of magnitude lower than that obtained by a conventional electrochemical measurement with the same electrode. The glucose can be selectively detected in the presence of interferences, such as ascorbic acid and uric acid, when the gate electrode is modified with Nafion. Moreover, the device shows a good stability and reproducibility. The OECTs will find broader applications in many other biosensors by modifying TNTAs gate electrodes with other enzymes and nanomaterials.

\section{Photoelectrochemical COD sensor with Pt- NPs/TNTAs as the gate electrode}

Another highly sensitive OECT-based sensor was developed for COD determination in wastewaters with a Pt-NPs/TNTAs gate electrode which could be activated by UV light [4]. The sensing mechanism can be contributed to the photoelectrochemical reaction of organic compounds on the gate electrode, which leads to the change of effective gate voltage applied on the transistor. The OECT devices show low detection limit of $0.01 \mathrm{mg} / \mathrm{L}$ COD, which is two orders of magnitude better than that of the conventional photoelectrochemical method using the same Pt-NPs/TNTAs electrode. The validity of the OECT devices for COD analysis has also been demonstrated by correlating the COD values of several types of real samples obtained from the OECT devices and the standard dichromate methods. Importantly, such OECT-based sensors combine the advantages of the organic thin film transistors (e.g., cost-effective, miniaturization, sensitivity), and those of the conventional photoelectrochemical method (e.g., stability, accuracy, fast response), while eradicate the latter's drawbacks of the large and clumsy three-electrode system.

\section{Conclusions and future outlook}

In summary, three high-performance OECTbased sensors with various gate electrodes have been presented for the applications in chemical and biological sensing. Various types of sensors, including diatom, glucose and COD sensors have been developed with promising properties using $\mathrm{Au}$, TNTAs and Pt-NPs/TNTAs as the gate electrodes, respectively. These OECT-based sensors have shown excellent performances in aqueous environment and thus great potentials for real-time in situ measurement. Since the materials selection, nanostructure control and surface modification could be easily achieved for the gate electrodes, these will facilitate the further research and development of OECT-based sensors for real applications.

\section{References}

[1] P. Lin, F. Yan, Organic Thin-Film Transistors for Chemical and Biological Sensing, Advanced Materials 24, 34-51 (2012); doi: 10.1002/adma.201103334

[2] J. Liao, S. Lin, K. Liu, Y. Yang, R. Zhang, W. Du, $\mathrm{S}$. Li, Organic electrochemical transistor based biosensor for detecting marine diatoms in seawater medium, Sensors and Actuators $B$ : Chemical 203, 677-682 (2014); doi: 10.1016/j.snb.2014.07.052

[3] J. Liao, S. Lin, Y. Yang, K. Liu, W. Du, Highly selective and sensitive glucose sensors based on organic electrochemical transistors using TiO2nanotube arrays-based gate electrodes, Sensors and Actuators B: Chemical 208, 457-463 (2015); doi: 10.1016/j.snb.2014.11.038

[4] J. Liao, S. Lin, M. Zeng, Y. Yang, A miniature photoelectrochemical sensor based on organic electrochemical transistor for sensitive determination of chemical oxygen demand in wastewaters, Water Research 94, 296-304 (2016); doi: 10.1016/j.watres.2016.02.061 